\title{
On the Initial Mass Function of Be Stars and the Missing Be Stars of Late Spectral Types
}

\section{J. Zorec}

Institut d'Astrophysique de Paris, CNRS, $98^{\mathrm{bis}}$ bd. Arago, F-75014 Paris, France

\begin{abstract}
This is an attempt at determining the IMF (Initial Mass Function) of Be stars relative to that of $B$ stars in the vicinity of the Sun. We have represented the IMF as a power function of the stellar mass $M^{\beta}$, so that the relative IMF is then proportional to $M^{\beta(\mathrm{Be})-\beta(\mathrm{B})}$. Allowing for systematic differences in the counting of $\mathrm{Be}$ stars due to their apparent overluminosity, a difference $\beta(B e)-\beta(B) \sim 0$ is found, which may indicate that there are no huge intrinsic differences between the two types of objects. In these calculations changes of spectral types due to high rotation were not taken into account. This effect may still strongly affect the results obtained. By extrapolating the $\left.M^{\beta} \mathrm{Be}\right)-\beta(\mathrm{B})$ curve to spectral types later than $\mathrm{B} 7$, we reckon that in a volume limited to the apparent magnitude $V=7$ there may be about 150 still undetected $\mathrm{Be}$ stars of late spectral types.
\end{abstract}

\section{Introduction}

Several ways by which a star can present a Be phenomenon are currently proposed. In a study of the frequency of Be stars in different luminosity classes, Zorec and Briot (1997) conclude that the Be phenomenon may arise due to innate properties in stars since their formation. This implies that the IMF of $\mathrm{Be}$ stars might have some differences as compared to that of other B stars. Zinnecker (1989) showed that the initial conditions of stellar formation may determine the characteristics of the IMF - frequency of stellar masses at birth. The aim of this paper is then to discover whether the present data on Be stars may lead to some indications that the peculiarities relative to Be stars are due to distinctive initial conditions and/or to processes involved in the stellar formation phases.

\section{The stellar sample}

Concerning the statistical completeness of stellar samples, at the moment the best sample of Be stars is the magnitude-limited one obtained from counting in the Bright Stars Catalogue (BSC, Hoffleit 1982) and Supplement (SBSC, Hoffleit et al. 1983). The sample of stars in these catalogues is sufficiently bright so that almost all $\mathrm{B}$ stars showing the Be phenomenon have already been detected. Nevertheless, Coté's et al. (1993) results show that we should not exclude the possibility of $\mathrm{B}$ stars still remaining with undetected Be phenomena. Thus, 


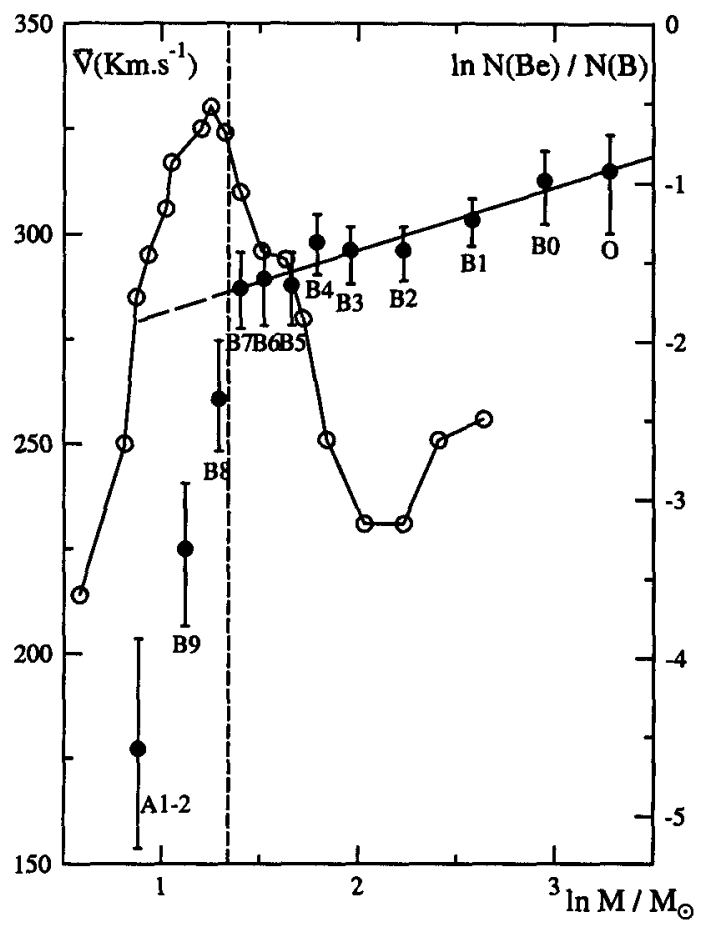

Figure 1. "•": Magnitude limited counts of Be relative to B stars as a function of stellar mass. "o" : Distribution of mean true rotational velocities of main sequence Be stars.

without taking into account the missing Be stars, we are left with a magnitudelimited sample of $325 \mathrm{Be}$ stars.

The sample of "B stars" is made up of stars currently considered as normal $B$ stars and of particular ones: metallic, helium, magnetic Bp stars and special types such as $\beta$ Cephei stars. Our $O$ type stars are in the spectral type interval O7-09.5. The A type stars are in the A0-A2 spectral interval. To estimate the sampling uncertainty, we transformed the "observed" magnitude-limited observed sample into a volume-limited one, using the spectral type-luminosity class dependent filling factor of samples derived by Zorec and Briot (1997). The sampling uncertainties thus obtained at a $95 \%$ confidence level are shown as error bars in Fig 1.

\section{Preliminary results}

For a preliminary search for possible differences between the IMF of $\mathrm{B}$ and Be stars, we assume that: (a) the distribution in space of Be stars mirrors that of other B stars; (b) the relation between the visible absolute magnitude and mass is the same for both types of objects; (c) the main sequence lifetime of $B$ 
and Be stars is the same; (d) the star formation rate is constant for each class of objects. These simplifications imply that the ratio between the IMF of B and $\mathrm{Be}$ stars is given by the ratio of the respective present day mass functions, which are proportional to counts of the respective stars in the main sequence (Scalo 1986, Rana 1987). We can then represent the behaviour of the IMF for stars with masses $3 \lessgtr \mathcal{M} \lesssim 20$ ( $\mathcal{M}$ is the stellar mass in solar units) as $\Psi(\mathcal{M})=\mathrm{d} \dot{N} / \mathrm{d} \ln \mathcal{M} \propto \mathcal{M}^{-\beta}$. So, the slope $\Delta \beta=\beta(\mathrm{B})-\beta(\mathrm{Be})$ of the curve $\ln \Lambda_{\Psi}=\ln [\Psi(\mathcal{M} \mid \mathrm{Be}) / \Psi(\mathcal{M} \mid \mathrm{B})] \propto \ln N(\mathrm{Be}) / N(\mathrm{~B})$ against $\ln \mathcal{M}$ can give us a first insight into the existence of possible differences between the IMF of $B$ and Be stars. The $\ln N(\mathrm{Be}) / N(\mathrm{~B})$ vs $\ln \mathcal{M}$ of main sequence $\mathrm{B}$ and $\mathrm{Be}$ stars is shown in Fig. 1. The regression line concerns stars of spectral types B0 to B7 only. In fact, counts of Oe stars are very uncertain and those of Be stars latter than B7, as we shall see, are probably underestimated.

Taking into account the sampling uncertainties, the slope of the line in Figure 1 is $\Delta \beta=0.43 \pm 0.03$. This difference may then imply that processes, or initial conditions, intervening in Be star formation are in some way different from those of other B stars with similar masses. However, counting of Be stars is affected by a number of biases due to their spectral and photometric peculiarities and to effects due to their high rotation. We shall see that the results obtained can be greatly modified when these effects are taken into account.

\section{Effect due to the overluminosity of Be stars}

Be stars present a flux excess due to the presence of the circumstellar envelope. So, on average they can be brighter than B stars without emission of the same mass. As we are dealing with magnitude limited samples of stars, Be stars are systematically counted in larger volumes of space than B stars without emission. The correction needed to reduce the counts of both groups of objects to the same volume in each spectral type was discussed in Zorec \& Briot (1997). According to this result, counts of Be stars need to be multiplied by a factor $\eta$ given by:

$$
\eta \sim 1.3 \times \mathcal{M}^{-0.3}
$$

Due to $\eta$, the difference between the IMF slopes of $\mathrm{Be}$ and that of remaining $\mathrm{B}$ stars reduces so that $\Delta \beta \rightarrow 0.0$. However, the estimation of the excess produced by the circumstellar envelope of $\mathrm{Be}$ stars in the $V$ magnitude is still subject to a number of uncertainties. In particular the effect derived by (1) may be overestimated. On the other hand, systematic effects due to the rapid rotation of Be tend to compensate the decrease of $\Delta \beta$ produced by $\eta$.

\section{The missing Be stars of late spectral types}

The regression line of Fig. 1 leads to the relation:

$$
(N(\mathrm{Be}) / N(\mathrm{~B}))_{\mathrm{dwarfs}} \simeq(0.10 \pm 0.03) \mathcal{M}^{(0.43 \pm 0.03)}
$$

Comparing these ratios to those obtained from counts, we can estimate the number of Be stars missing in the later spectral types than B7 to recover the 
slope (1). This leads to the conclusion that there may be about 150 still unknown main sequence Be stars in the spectral types interval from B8 to A2. On the other hand, knowing that high rotation is one of the most outstanding characteristics of Be stars, and that precisely among late Be stars there is an abrupt increase of the mean $V \sin i$, the presence of a significant fraction of still unknown Be stars should not be surprising in principle. In Fig. 1 we also show the distribution of mean values of true rotational velocities $\bar{V}=(4 / \pi) \overline{V \sin i}$ of main sequence Be stars derived from Zorec et al. 1990, which clearly shows the increase of $\bar{V}$ among the latest B spectral types. Recently Ghosh et al. (1999) discovered six new Be stars of late spectral types.

\section{References}

Coté, J., van Kerkwijk, M.H. 1993, A\&A 274, 870

Garmany, C.D., Conti, P.S. 1982, ApJ 263, 777

Ghosh, K.K., Apparao, K.M.V., Pukalenthi, S. 1999, A\&AS 134, 358

Hoffleit, D., Jaschek, C. 1982, The Bright Star Catalogue, Yale Univ. Observatory (BSC)

Hoffleit, D., Saladyga, M., Wlasuk, P. 1983, A Supplement to the Bright Star Catalogue, Yale Univ. Observatory (SBSC)

Rana, N.C. 1987, A\&A 184, 104

Scalo, J.M. 1986, Fundamentals of Cosmic Physics 11, 1

Zinnecker, H. 1989, Towards a Theory of Star Formation. In: Beckman J.E., Pagel B.E.J. (eds.), Evolutionary Phenomena in Galaxies. Cambridge University Press, p. 113

Zorec, J., Briot, D. 1997 A\&A 318, 443

Zorec, J., Mochkovitch, R., Garcia, A. 1990, The Angular Momentum-Loss and the Differential Rotation in B and Be Stars. In: Willson L.A., Stalio R. (eds.) NATO ASI Series C, Vol. 316, Angular Momentum and Mass Loss for Hot Stars, Kluwer Academic Publishers, p. 239 